FORMATION Formation emploi

Revue française de sciences sociales

130 | Avril-Juin 2015

L'essor des compétences non académiques

\title{
Post-face : Retour sur le concept de compétences non académiques
}

Franck Bailly et Alexandre Léné

\section{(2) OpenEdition \\ Journals}

Édition électronique

URL : http://journals.openedition.org/formationemploi/4419

DOI : 10.4000/formationemploi.4419

ISSN : 2107-0946

Éditeur

La Documentation française

Édition imprimée

Date de publication : 10 juin 2015

Pagination : 69-78

ISSN : 0759-6340

Référence électronique

Franck Bailly et Alexandre Léné, « Post-face : Retour sur le concept de compétences non académiques », Formation emploi [En ligne], 130 | Avril-Juin 2015, mis en ligne le 08 juin 2017, consulté le 30 octobre 2020. URL : http://journals.openedition.org/formationemploi/4419; DOI : https://doi.org/10.4000/

formationemploi.4419

(c) Tous droits réservés 


\title{
Postface
}

\section{Retour sur le concept de compétences non académiques}

\begin{abstract}
FRANCK BAILLY
Maître de conférences en économie, CREAM (Centre de recherche en économie appliquée à la mondialisation) - EA 4702. UFR de droit, sciences économiques et gestion, université de Rouen
\end{abstract}

AleXANDRE LÉNÉ

Maître de conférences en économie, CLERSE (Centre Lillois d'études et de recherche sociologiques et économiques) CNRS, Faculté des sciences économiques et sociales, université

de Lille 1

Le concept de compétences non académiques - celles-ci se déclinant également dans la littérature sous les termes de compétences soft, de compétences non cognitives ou encore de traits de personnalité ou de compétences socio-émotionnelles (Heckman et Kautz, 2012) - est au cœur de la dynamique des économies contemporaines. Il occupe une place croissante dans les travaux anglophones, qu'ils soient économiques, sociologiques, psychologiques ou liés aux sciences de gestion. Une importance moindre semble lui être accordée en France. Contribuant à combler cette lacune, l'initiative de Formation Emploi de consacrer un dossier aux compétences non académiques est donc à saluer.

Lintérêt des trois articles du dossier est, tout à la fois, de prendre au sérieux la question des compétences non académiques et d'aborder de front les nombreux problèmes qu'elle pose. Ces problèmes peuvent être appréhendés à différents niveaux.

Les questions sont tout d'abord d'ordre scientifique : comment définir, appréhender, mesurer et analyser la montée de ces compétences ?

Les difficultés sont également d'ordre opérationnel : comment les différents acteurs économiques, notamment les organisations, repèrent-ils ces compétences ? Quelles sont les difficultés rencontrées et quels sont les moyens mis en ouvre pour les produire, les reconnaître, les évaluer ? La montée des compétences non académiques se traduitelle par la construction d'outils particuliers?

Enfin, les articles du présent numéro révèlent bien les enjeux sociaux de ce phénomène, particulièrement en ce qui concerne le fonctionnement du système éducatif. Nous reprenons successivement chacun de ces thèmes. 


\section{Les compétences non académiques: la montée d'une notion complexe qui fait débat}

\subsection{L'essor des compétences non académiques}

L'intérêt des chercheurs pour le concept de compétences non académiques soulève d'emblée une question : s'agit-il d'une sorte d'effet de mode qui viserait à nommer différemment une réalité qui existait déjà ou l'émergence du concept renvoie-t-elle à un changement plus profond de la réalité économique et sociale ?

Les travaux visant à évaluer l'importance de ces compétences semblent s'accorder sur un certain nombre de constats qui tendraient à conforter la seconde option.

Depuis une trentaine d'années, en effet, les économies développées ont connu des mutations importantes. Le développement de nouvelles technologies, l'émergence de relations professionnelles de plus en plus influencées par la présence du client dans le processus de production ont remodelé les modes d'organisation du travail. Parallèlement, les économies se sont fortement tertiarisées. Ces changements ont engendré une demande accrue de compétences telles que la capacité à résoudre des problèmes, à établir des diagnostics, à communiquer, de compétences sociales et interpersonnelles, de compétences émotionnelles,... bref, de compétences non académiques.

Différentes études ont cherché à mesurer l'importance et la montée de ces compétences non académiques. Par exemple, Green et al. (1998) ont tenté d'appréhender ces nouvelles exigences en interrogeant un échantillon représentatif de 1693 employeurs britanniques en leur demandant de citer selon eux, les " qualités critiques " de leurs salariés, c'est-à-dire les qualités qui leur font défaut et qu'ils devraient "idéalement " posséder : manque de compétences techniques, manque d'expertise, manque de compétences de base, problème d'attitude, de motivation, problèmes liés à la personnalité, manque d'expérience... Il ressort de leur analyse qu'un établissement sur deux cite comme importantes les attitudes, la motivation et la personnalité. Les compétences relationnelles et interpersonnelles sont jugées nécessaires dans toutes les entreprises. Ce sont des compétences jugées critiques, surtout dans les emplois non manuels.

Borghans, ter Weel et Weinberg (2008) montrent quant à eux que les changements organisationnels et techniques ont accru l'importance des compétences interpersonnelles (être capable de s'adapter aux sentiments des autres, de s'identifier aux autres, de communiquer avec les autres sans les contrarier, influencer les autres), même si cette importance varie fortement d'un emploi à l'autre. Le secteur des services est bien sûr particulièrement marqué par l'exigence de compétences non académiques. Il n'est d'ailleurs pas surprenant que les débats autour de ces compétences (appréhendées en termes de soft skills) aient été initiés en Grande-Bretagne, dont l'économie en voie de désindustrialisation était caractérisée par une montée des emplois tertiaires (Payne, 2004). 


\subsection{Une notion difficile à saisir et qui suscite des débats}

Appréhender et mesurer les compétences non académiques n’est pas aisé. Cette notion est difficile à définir et variable selon les auteurs. La diversité des termes utilisés dans la littérature, que nous rappelions en introduction, l'illustre.

Cette diversité se retrouve également dans l'ensemble des articles du dossier. La nature des qualités que l'on peut ranger sous ce terme est en effet très diverse.

La revue de la littérature (non exhaustive) que propose Damien Collard sur le thème des compétences de service souligne combien les approches possibles sont diverses et variées. Des travaux en sociologie, en ergonomie et en sciences du langage principalement, ont permis depuis longtemps de mettre en évidence "l'extension et la variété » des registres de compétences des agents au contact du public. Ainsi, la notion de compétence a non seulement gagné tous les milieux (entreprise, formation, école) mais son "domaine " n’a cessé de s'étendre.

On distingue ainsi les compétences relationnelles (capacité à communiquer, mais également des qualités personnelles des employés comme l'enthousiasme, la convivialité), les compétences de gestion de l'interaction (aptitude à se mettre à la place de l'autre, capacité à pacifier les relations avec les clients), les compétences d'organisation (mobiliser d'autres compétences que les siennes propres pour aboutir à une solution), les capacités à gérer ses émotions et à faire preuve d'empathie, les attitudes (responsabilité, ouverture d'esprit, adaptabilité, tolérance, confiance en soi, envie d'apprendre), voire les compétences esthétiques (transmettre une image satisfaisante, en cohérence avec l'image souhaitée par l'organisation)... A cela s'ajoute la diversité des registres auxquels peuvent être rattachées les compétences non académiques : capital social, attitudes, prédispositions ou traits de personnalité. Au final, il se dégage, comme le souligne Marie Duru-Bellat, une sorte de " halo" autour du concept de compétences non académiques. Ce flou intrinsèque à la notion pose évidemment des problèmes au chercheur qui souhaite l'appréhender.

Ce flou se trouve encore accentué par d'autres clivages qui traversent les analyses. C'est le cas de la diversité des champs disciplinaires ou écoles de pensées auxquels appartiennent les chercheurs.

Par exemple, certains reprochent au concept son côté normatif et son alignement sur le discours des employeurs (Grugulis et Stoyanova, 2011). Ils voient dans les compétences non académiques un moyen d'interroger la relation de pouvoir entre les employeurs et les salariés, alors que dans le même temps ce concept peut être utilisé par des économistes qui n'ont pas la même lecture conflictuelle de la relation de travail.

Les économistes néo-classiques adoptent en effet une autre perspective, où chaque salarié négocie avec son employeur et valorise ses compétences dans le cadre d'un "bilatéralisme contractuel ". Cette même notion est ainsi utilisée au sein de para- 
digmes différents qui proposent des conceptions totalement différentes de la relation d'emploi.

Les caractéristiques et propriétés attribuées, parfois de façon implicite, aux compétences non académiques constituent une autre ligne de complexification. Certaines analyses assimilent les compétences non académiques aux traits de personnalité, alors que d'autres approches insistent sur leur plasticité, leur caractère apprenable. Heckman et Kautz (2012, p. 452) indiquent en effet que " les différents noms employés évoquent des propriétés différentes. Le terme "trait" suggère une idée de permanence et aussi un caractère possiblement héréditaire. Les termes "compétences" et "caractères" suggèrent quils peuvent être appris »'. Considérer que ces caractéristiques personnelles sont stables ou qu'elles ne le sont pas engendre des approches et des questionnements radicalement différents, qui peuvent amener à mettre l'accent, d'un côté sur les phénomènes de sélection et d'appariement, ou, de l'autre côté, sur la question de la formation par exemple.

Toutefois, le statut actuel du concept de compétences non académiques et les difficultés que l'on vient d'évoquer ne lui sont sans doute pas spécifiques. Du point de vue de l'histoire des idées, il s'agit probablement d'un processus commun à de nombreux concepts désormais bien acceptés. Tel est le cas, par exemple, d'une autre mesure de la compétence qu'est le concept de capital humain, qui s'est imposé non sans certaines réserves ou tensions (Teixeira, 2000 ; 2005). Pour autant, ces difficultés ne doivent pas, nous semble-t-il, masquer les enjeux liés à l'essor des compétences non académiques.

\section{La montée des compétences non académiques: enjeux pour les organisations et la société}

De façon schématique, ces enjeux peuvent concerner le mode de fonctionnement des organisations, notamment des entreprises, ou avoir une portée sociale plus large.

\subsection{Des enjeux pour les organisations...}

\subsubsection{Articuler compétences non académiques et compétences académiques}

Comment s'articulent les compétences non académiques et les compétences académiques? Les premières ont-elles remplacé les secondes ou y a-t-il, au contraire, complémentarité ? L'article de François Sarfati permet d'éclairer cette question, sous l'angle de la sociologie de l'éducation, en révélant comment les compétences exigées pour intégrer le monde du travail à la suite d'une formation professionnalisante relèvent à la fois du registre académique

1. "These different names connote different properties. The term " traits" suggests a sense of permanence and possibly also of heritability. The terms "skills" and "character " suggest that can be learned" 
et non académique. Les travaux économiques que recensent Balcar (2014) vont également dans le même sens : il existerait une corrélation positive entre les exigences des entreprises en compétences académiques et non académiques.

\subsubsection{La montée des compétences non académiques affecte les comportements des organisations}

Le deuxième enjeu concerne la manière dont les comportements des organisations, notamment des entreprises, sont affectés par la montée des compétences non académiques. Au moins deux facettes de ces comportements doivent être distinguées : l'une relative à la reconnaissance des compétences non académiques, et l'autre à la sélection et à la discrimination.

En ce qui concerne la question de la reconnaissance des compétences non académiques. l'idée selon laquelle ces compétences semblent être reconnues et récompensées en termes de salaire est ancienne. Elle est au cœur des travaux critiques, bien que rarement cités, des économistes radicaux des années 1970 (Gintis, 1971 ; Edwards, 1976, 1977) et semble être confirmée par les analyses récentes ( $c f$. par exemple, Bowles, Gintis et Osborne, 2001 ; Balcar, 2014).

Mais la reconnaissance par les organisations, et particulièrement les entreprises, ne s'arrête pas aux questions de salaire, comme le rappelle l'article de Damien Collard. Les compétences non académiques sont largement tacites, insaisissables et difficiles à objectiver, ce qui n'est pas sans rappeler l'écart pointé par la sociologie du travail entre le travail réel et le travail prescrit. Damien Collard montre alors comment l'instrumentation gestionnaire se révèle souvent inadaptée et ne permet pas de reconnaître pleinement les compétences non académiques détenues par les acteurs. Les outils de GRH (gestion des ressources humaines) utilisés en interne (que ce soit le livret de professionnalisation, les fiches de postes ou les référentiels de compétences qui servent de support d'évaluation) " écrasent » littéralement le travail réel et une partie des compétences mises en jeu dans l'exercice de l'activité. Il y a, pour reprendre l'expression évocatrice de l'auteur, des " angles morts dans l'instrumentation gestionnaire".

Il s'agit là d'une réelle difficulté. En effet, la reconnaissance institutionnelle des compétences professionnelles passe nécessairement par les outils pour gérer les carrières, organiser les recrutements et les mobilités, ou encore rémunérer les personnes. Autant d'actes de gestion qui devraient reposer sur des critères objectivés. La non-prise en compte des compétences non académiques, rappelle Damien Collard, peut générer amertume, ressentiment et finalement, pourrions-nous ajouter, moindre implication des salariés.

Au-delà du cas étudié par l'auteur, la montée des compétences non académiques s'inscrit, plus largement, dans une tendance à l'abandon des formes antérieures de la régulation sociale qui prenaient appui sur la logique de qualification (Bailly et Léné, 2013). Se trouve alors posée la question de l'institutionnalisation des formes de reconnaissance 
de ces compétences, notamment dans les activités en émergence ou celles qui ne sont pas encore stabilisées du point de vue de la professionnalité des métiers qui y sont exercés. Cette question nous semble particulièrement vive dans les métiers de services qui ont toujours peiné, historiquement, à formaliser des systèmes de classifications clairs et structurés.

La seconde facette du comportement des organisations concernée par la montée des compétences non académiques est relative aux questions de sélection et de discrimination. En effet, les compétences non académiques ne sont pas toujours faciles à repérer et en particulier au moment du recrutement. Il existe une vaste littérature sur ce sujet. Pour évaluer ces compétences, les recruteurs peuvent adopter deux attitudes opposées : soit multiplier les critères et les preuves, soit, au contraire, basculer dans le registre de l'intuition et du non-formalisé (de Larquier et Marchal, 2012).

Dans le premier cas de figure, les entreprises peuvent avoir recours à des techniques parfois très sophistiquées pour identifier les meilleurs candidats : observation in situ des comportements, jeux de rôles et tests de personnalité (Morris et Feldman, 1997). Les procédures de recrutement sont alors complexes et comportent de nombreuses étapes. C'est par exemple le cas pour le centre d'appels d'une banque, qu'évoquent Thompson, Warhurst et Callaghan (2001), où les recruteurs évaluent les candidats dans trois domaines: les traits de caractère, les compétences relationnelles (communication orale surtout) et les compétences techniques (manipulation du clavier, lecture des chiffres). La multiplication de ces épreuves viserait à limiter les incertitudes qui sont toutefois de natures très variables. Elles peuvent être très formalisées, mais sont souvent plus informelles (simple entretien individuel). Dans ce deuxième cas de figure, les employeurs peuvent ainsi utiliser leur "feeling" pour émettre des jugements sur les compétences des candidats à l'embauche. C'est notamment le cas dans la grande distribution, analysée par Rieucau et Salognon (2013). Lors du recrutement, les compétences s'évaluent lors d'entretiens qui peuvent être très courts. Le recruteur mobilise ses intuitions et ses impressions sans qu'aucune véritable règle ne soit explicitée. Bien qu'elles soient constamment mobilisées et mises en avant par les managers pour justifier leurs décisions, les qualités relationnelles, comportementales et psychologiques des salariés restent des notions floues, de sorte que les jugements renvoient en fait à la logique du bon sens et à l'intuition, c'est-à-dire à l'univers du non-formalisé.

La sélection et le repérage de compétences non académiques ne sont pas limités à la sphère de l'entreprise. Ils sont également présents dans les filières de formation sélectives, notamment celles qui sont professionnalisées. L'article de François Sarfati montre que la logique sous-jacente n'est pas totalement différente. Bien sûr, plus les pratiques s'appuient sur des éléments comme la présentation, l'attitude, le comportement, plus elles sont susceptibles de se fonder sur des stéréotypes en termes de classe sociale, d'origine ou de genre et d'engendrer des comportements discriminatoires (Tilly et Moss, 1996 ; Payne, 2004). C’est ce que souligne également la contribution de Damien Collard. 


\subsubsection{La place de la formation dans le développement des compétences non académiques}

Le troisième enjeu a trait à la question de la formation. Les organisations peuvent en effet souhaiter développer les compétences non académiques de leurs salariés par la formation. Mais le caractère tacite de ces compétences rend difficile leur prise en charge par les acteurs traditionnels de la formation. De nouvelles pratiques de formation sont toutefois observées. La contribution de Damien Collard, qui rapporte l'expérience menée au sein de la SNCF pour détecter et développer les compétences de service, est tout à fait éclairante. Des ateliers d'improvisation théâtrale, des mises en situation, des jeux de rôle filmés, l'utilisation de la PNL (programmation neuro-linguistique) et de l'analyse transactionnelle sont mobilisés pour aider les agents à adopter les comportements adaptés aux situations.

De telles stratégies ne sont pas sans limites. Il y a bien sûr celle évoquée précédemment : la tendance des organisations à écraser les compétences non académiques et donc à ne pas les reconnaître. Mais il faudrait également ajouter la croyance de certains employeurs dans le caractère inné ou naturel de ce type de compétences, qui n’incite pas non plus à investir dans la formation. De plus, lorsque ces qualités sont jugées comme pouvant être apprises, la responsabilité peut être reportée sur d'autres acteurs comme l'école ou la famille (Bailly et Léné, 2009 ; 2013).

\section{2... mais également des enjeux sociaux}

Il existe également des enjeux sociaux relatifs à la montée des compétences non académiques. Cela apparait clairement s'agissant des risques de discrimination évoqués précédemment. Mais au-delà, au moins deux autres enjeux sociaux sont à relever.

\subsubsection{Un nouveau regard sur la réalité sociale et économique}

Les compétences non académiques conduisent à porter, sur la réalité sociale et économique, un regard qui n'est pas neutre. Il n'est pas neutre, d'une part, en ce qui concerne le contenu de ces compétences. En effet, comme le note Marie Duru-Bellat, «si les compétences sont ce qui permet d'être évalué positivement, elles nous renvoient une image de ce que la société valorise à l'instant t $»$. D'autre part, ce regard n'est pas neutre non plus s'agissant du concept lui-même. De nombreux travaux, notamment en sociologie du travail, ont ainsi insisté sur le processus de construction sociale du concept de compétences non académiques (Dubernet, 1996 ; Kergoat, 2007). Or, sans doute plus encore que celui de compétences " en général " (Lallement, 2007), le concept de compétences non académiques, parce qu'il fait référence aux caractéristiques des personnes, s'inscrit dans une logique de l'individualisation de la relation salariale qui peut tendre à rendre moins visible le lien de subordination.

Cet accent mis sur l'individu comme clé de lecture des phénomènes économiques peut apparaître comme un curieux retournement de l'histoire. La notion de compétences non 
académiques introduite, on l'a rappelé, par les économistes radicaux dans les années 1970 $s$ 'inscrivait, en effet, dans une perspective critique. Leur objectif était de montrer comment cette demande de compétences (qui s'exprime dans leurs travaux en termes de personnal traits) reflétait la volonté des employeurs de mieux contrôler les salariés. Loin d'affirmer une lecture individualiste de la relation salariale, il s'agissait, au contraire, d'en rappeler son caractère conflictuel (Bailly et Léné, 2015).

\subsubsection{Quel impact sur la reproduction des inégalités sociales ?}

Le deuxième enjeu découle, en partie, du précédent. Il concerne l'éducation. L'article de Marie Duru-Bellat rappelle que la sociologie de l'éducation et une partie des travaux en économie de l'éducation (Bowles et Gintis, 1976) des années 1970 avaient pointé le rôle de l'école dans la reproduction des inégalités sociales. La montée des compétences non académiques ne relègue pas au second plan la question de ces inégalités. Bien au contraire. C'est ce que rappelle la contribution de François Sarfati. Pour pouvoir accéder à la filière de formation qu'il étudie, un master professionnalisé par voie d'apprentissage, la sélection est intense. Elle repose non seulement sur une évaluation des compétences scolaires, mais également sur des compétences professionnelles non académiques que les candidats doivent mettre en scène dans les entretiens de recrutement. Les trajectoires sociales et professionnelles, l'attitude, les apparences vestimentaires, l'élocution sont autant de signaux utilisés comme révélateurs des compétences attendues. Le risque est grand alors de favoriser ceux qui ont le moins besoin de réaliser un parcours professionnalisant en entreprise et ainsi de permettre à celles et ceux qui en sont déjà bien dotés d'acquérir des compétences professionnelles; ce qui revient à évincer les titulaires de compétences scolaires qui auraient justement le plus besoin de confronter leurs savoirs académiques aux situations professionnelles. Ce n'est pas le moindre des paradoxes d'une formation professionnalisante.

Au-delà de ce cas, il s'agit, plus largement, à travers la formation, conclut Marie DuruBellat, d' " apprendre à tous à décrypter ce qui fait, au jour d'aujourd'hui et dans tel contexte, le 'mérite professionnel'", au risque sinon que cela soit "de fait, le privilège des 'héritiers" ". Car on peut en effet considérer qu'une partie des compétences non académiques ont été acquises par les individus au cours de leur socialisation primaire et qu'elles relèvent du capital culturel et social (Pettinger, 2004).

\section{Conclusion}

Le tour d'horizon que nous proposons à travers la lecture de ces trois contributions ne prétend pas épuiser la complexité du sujet. D’autres pistes pourraient être explorées.

Nous pouvons en suggérer quelques-unes : d'une part, les compétences non académiques s'inscrivent-elles pleinement dans ce qu'il est convenu d'appeler " la logique compétence " ? Autrement-dit, n'y a-t-il pas, notamment dans les pratiques des entreprises, au sein de la logique compétence, des spécificités liées aux compétences non académiques? 
D'autre part, l'analyse des compétences non académiques ne devrait-elle pas être menée plus finement au niveau du type d'emploi ou de métiers concernés ? Pour le dire un peu rapidement, les compétences non académiques mobilisées au bas de l'échelle hiérarchique sont-elles de même nature que celles nécessaires pour les postes les plus élevés, quand bien même il serait possible de les qualifier de la même façon (par exemple, capacité à communiquer, attention à autrui,....) ? Enfin, un travail resterait sans doute encore à réaliser pour comprendre comment les différents partenaires sociaux, et notamment les syndicats, s'emparent de la question des compétences non académiques. Autant dire que le chantier est encore vaste.

\section{Bibliographie}

Balcar J. (2014), "Soft Skills and their Wage Returns: Overview of Empirical Literature”, Review of Economic Perspectives, 14, pp. 3-15.

Bailly F., Léné A., Toutin M.-H. (2009), « La portée formatrice de l'expérience : les employeurs du secteur des services ", Formation Emploi, 106, pp. 41-58.

Bailly F., Léné A. (2013), “The personification of the service labour process and the rise of soft skills: a French case study", Employee Relations, 35, pp. 79-97.

Bailly F., Léné A. (2015), "What makes a good worker? Richard Edwards is still relevant", Review of Radical Political Economics, à paraître.

Borghans L., ter Weel B., Weinberg B. A. (2008), "Interpersonal Styles and Labor Market Outcomes”, Journal of Human Resources, 43, pp. 815-58.

Bowles S., Gintis H.? Osborne M. (2011), "Incentive-Enhancing Preferences: Personality, Behavior, and Earnings", American Economic Review, 91, pp. 155-158.

Bowles S., Gintis H. (1976), Schooling in Capitalist America: Educational Reform and the Contradictions of Economic Life, New York: Basic Books.

de Larquier G., Marchal E. (2012), « La légitimité des épreuves de sélection : apports d’une enquête statistique auprès des entreprises ", in Eymard-Duvernay F. (Dir.), Epreuves d'évaluation et chômage, Toulouse, Octarès éditions, pp. 47-77.

Dubernet A.-C (1996), «La sélection des qualités dans l'embauche. Une mise en scène de la valeur sociale ", Formation Emploi, 54, pp. 3-14.

Edwards R. (1976), "Individual traits and organizational incentives: What makes a 'good' worker?”, Journal of Human Resources, 11, pp. 51-68.

Edwards R. (1977), "Personal traits and 'success' in schooling and work”, Educational and Psychological Measurements, 37, pp. 125-138. 
Gintis H. (1971), "Education, Technology, and the Characteristics of Worker Productivity", American Economic Review, 61, pp. 266-79.

Green F., Machin S., Wilkinson D. (1998), "The meaning and determinants of skills shortages", Oxford Bulletin of Economics \& Statistics, 60, pp. 165-87.

Grugulis I., Stoyanova D. (2011), "Skill and Performance", British Journal of Industrial Relations, 49, pp. 515-536.

Heckman J., Kautz T. (2012), "Hard evidence on soft skills", Labour Economics, 19, pp. 451-464.

Kergoat P. (2007), « Une redéfinition des politiques de formation. Le cas de l'apprentissage dans les grandes entreprises ", Formation Emploi, 99, pp. 13-27.

Lallement M. (2007), Le travail. Une sociologie contemporaine, Folio essais. 676 p.

Morris J. A., Feldman D. C. (1996), "The dimensions, antecedents, and consequences of emotional labor", Academy of Management Review, 21, pp. 986-1010.

Payne J. (2004), “The changing meaning of skill”, SKOPE Issues Paper $n^{\circ} 1$, University of Warwick, October.

Pettinger L. (2004), "Brand Culture and Branded Workers: Service Work and Aesthetic Labour in Fashion Retail”, Consumption, Markets \& Culture, 7, pp. 165-184.

Rieucau G., Salognon M. (2013), « Le recrutement dans la grande distribution : des pratiques ajustées ?", La revue de l"IRES, 76, pp. 45-69.

Teixeira P. N. (2000), "A Portrait of the Economics of Education, 1960-1997", History of Political Economy, Supplement, 32, pp. 257-87.

Teixeira P. N. (2005), "The 'Human Capital Revolution' in Economics", History of Economic Ideas, 13, pp. 129-48.

Thompson P., Warhurst C., Callaghan G. (2001), "Ignorant theory and knowledgeable workers: interrogating the connections between knowledge, skills and services", Journal of Management Studies, 38, pp. 923-942.

Tilly C., Moss P. (1996), "Soft Skills and Race: An investigation into black men's employment problems", Work and Occupations, 23, pp. 252-276. 